\title{
agNANO
}

\section{E-Beam Nanostructuring and Direct Click Biofunctionalization of Thiol-Ene Resist}

\author{
${ }_{3}$ Reza Zandi Shafagh, ${ }^{(1)}$ Alexander Vastesson, ${ }^{(0)}$ Weijin Guo, Wouter van der Wijngaart,*(1) \\ 4 and Tommy Haraldsson
}

5 KTH Royal Institute of Technology, Stockholm 10044, Sweden

6

Supporting Information

\begin{abstract}
Electron beam lithography (EBL) is of major importance for ultraminiaturized biohybrid system fabrication, as it allows combining biomolecular patterning and mechanical structure definition on the nanoscale. Existing methods are limited by multistep biomolecule immobilization procedures, harsh processing conditions that are harmful to sensitive biomolecules, or the structural properties of the resulting protein monolayers or hydrogel-based resists. This work introduces a thiol-ene EBL resist with chemically reactive thiol groups on its native surface that allow the direct and selective "click" immobilization of biomolecules under benign processing conditions. We constructed EBL structured features of size down to 20 $\mathrm{nm}$, and direct functionalized the nanostructures with a sandwich of biotin and streptavidin. The facile combination of polymer nanostructuring with biomolecule immobilization enables mechanically robust biohybrid components of interest for nanoscale biomedical, electronic, photonic, and robotic applications.
\end{abstract}

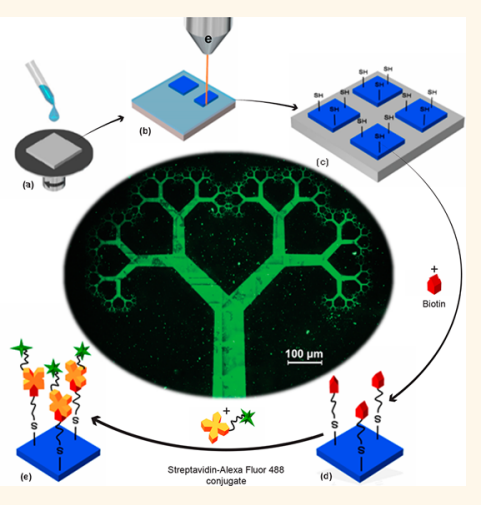

KEYWORDS: e-beam, thiol-ene, biohybrid, nanoscale

21
22
23 he emerging field of biohybrid systems combines biological and synthetic structural elements for biomedical or robotic applications. ${ }^{1,2}$ The constituting 24 elements of bionanoelectromechanical systems (BioNEMS) 25 are of nanoscale size, for example, DNA, proteins, or 26 nanostructured mechanical parts. This work reports the facile 27 top-down nanostructuring of polymers to create cross-linked 28 and mechanically robust nanostructures that are subsequently 29 functionalized with proteins.

30 Electron beam lithography (EBL)-based methods allow the 31 direct-write patterning of matter with sub-10 nm resolution. E32 beam patterning of protein monolayers, for example, by direct33 write inactivation of proteins, ${ }^{3}$ results in planar structures 34 unsuited as mechanical elements. One strategy toward 3D 35 biofunctionalized nanostructures is the incorporation of 36 proteins in hydrogel-based e-beam resists. Kolodziej and 37 Maynard ${ }^{4}$ review EBL-based patterning of biomolecules with 38 poly(ethylene glycol) (PEG)-based resists. However, these 39 methods suffer from either requirement of complex, multistep 40 surface modification or exposure of proteins to high vacuum 41 and electron radiation conditions not suited for molecules that 42 are prone to denaturation. Bat et al. ${ }^{5}$ describe hydrogel resists 43 of mixtures containing trehalose glycopolymer and proteins, 44 which allow direct-write patterning of multiple proteins by $45 \mathrm{EBL}$ while protecting the proteins from the harsh processing 46 conditions. However, whereas hydrogel resists might be of 47 interest for cell anchoring or cell proliferation applications, 48 they result in poor mechanical properties and make structuring 49 below $100 \mathrm{~nm}$ difficult.
A variety of different applications in organic electronics, 50 optics, and biomedicine utilize thiol-ene alternating copoly- 51 mers because of their tunable mechanical properties, low- 52 stress, low shrinkage, and fast curing process. ${ }^{6,7}$ Previous 53 nanostructuring of thiol-enes, using nanocontact molding, ${ }^{8} 54$ soft imprint lithography, ${ }^{9}$ step and flash imprint lithography, ${ }^{10}{ }_{55}$ or UV nanoimprint lithography, ${ }^{11}$ requires a cumbersome 56 master stamp or mold fabrication and suffers from residual 57 polymer layers and processing imperfections intrinsic to 58 contact-mode processes. ${ }^{12,13}$

Off-stoichiometric thiol-ene polymers (OSTE) ${ }^{14}$ feature 60 reactive thiol groups on their native surface, which can be 61 exploited for rapid one-step microscale surface patterning of 62 biomolecules. ${ }^{15}$ Previous OSTE-based devices were shown to 63 be biocompatible ${ }^{16}$ and suitable as a substrate for cell growth. ${ }^{17} 64$ The off-stoichiometric formulation also significantly reduces 65 pattern broadening during photostructuring due to diffusion- 66 induced monomer depletion. ${ }^{18}$

This work extends direct-write OSTE structuring from 68 photostructured microscale features to e-beam structured 69 nanoscale features with maintained surface reactivity, and 70 demonstrates the direct immobilization of biotin-streptavidin 71 complexes on the native nanostructured surfaces (see Figure 72 1).

Received: May 17, 2018

Accepted: September 13, 2018

Published: September 13, 2018 


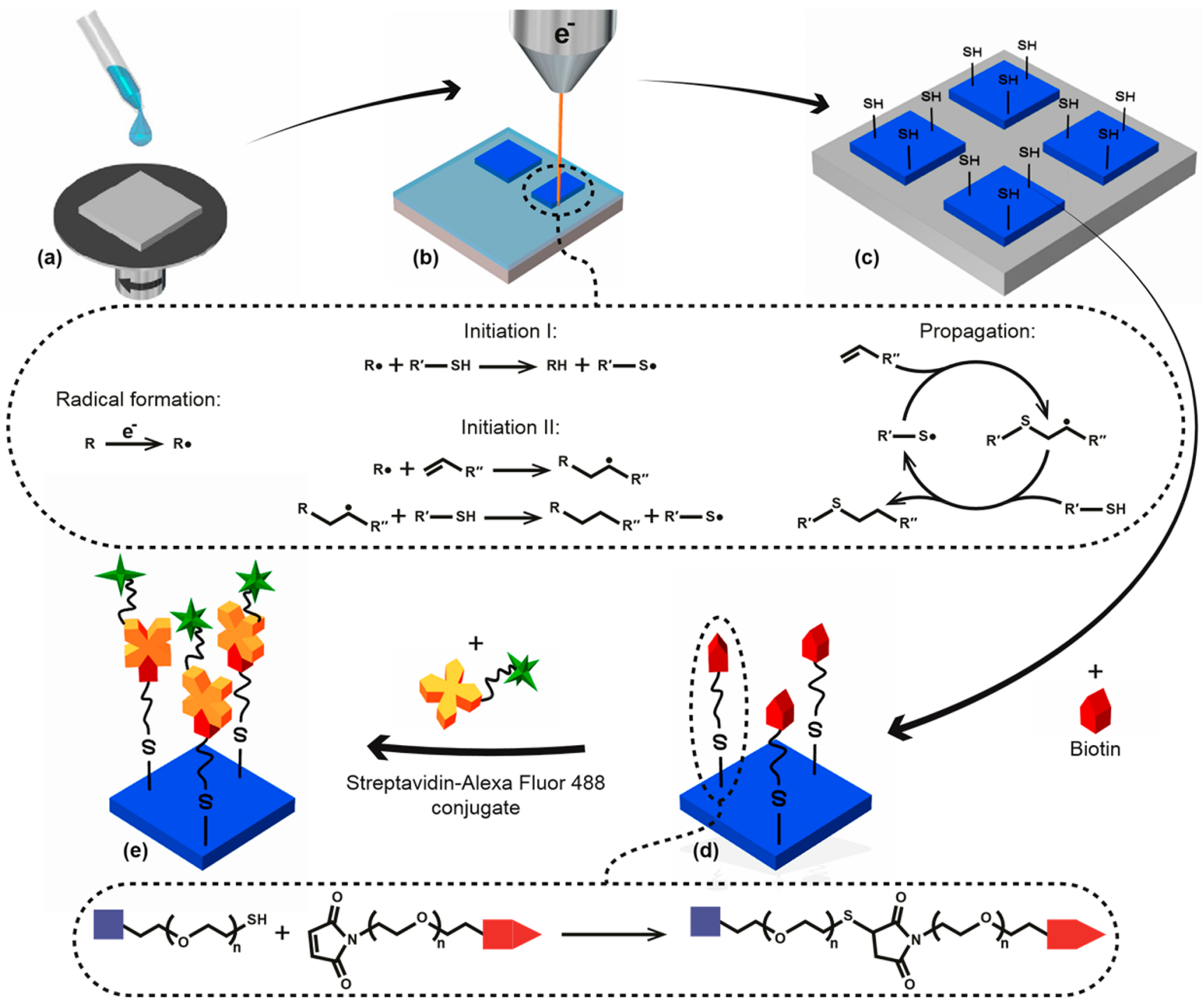

Figure 1. Schematic of the e-beam patterning and direct functionalization of OSTE. (a) Spin coating of OSTE resist. (b) E-beam curing reactions. (c) OSTE structures after patterning expose thiol functional groups on their surfaces. (d) Biotin linkers bind spontaneously to the unreacted thiols on the OSTE surface via thiol-maleimide Michael addition. (e) Fluorescently labeled streptavidin conjugate binds spontaneously to the biotin. EBL, e-beam lithography; OSTE, off-stoichiometric thiol-ene.

74 Thus, this work introduces three technical key advances. 75 First, direct nanostructuring of thiol-ene copolymers using ${ }_{76}$ EBL. Second, e-beam resist that features a chemically reactive 77 surface after patterning. Third, direct covalent (click) ${ }_{78}$ biofunctionalization of e-beam resist. The importance of our 79 method lies in the nature of the thiol functionality and its 80 spatial control. Thiol-click chemistry in combination with 81 nanostructuring can be used to achieve an outstanding control 82 of bottom up chemical synthesis, ${ }^{19}$ biomolecule immobiliza83 tion, ${ }^{15,20}$ and nanoscale self-assembly ${ }^{21}$ in a geometrically ${ }_{84}$ highly controlled manner. Due to the nature of thiol-click ${ }_{85}$ chemistry most processes are performed under benign 86 conditions $^{6,19,22}$ that are compatible with proteins, ${ }^{15,20}$ nucleic 87 acids, $^{23}$ and cell cultures. ${ }^{17,24,25}$ The facile structuring and 88 biomolecule immobilization is important for building bio89 nanoelectromechanical structures (Bio-NEMS) that can be ${ }_{90}$ implemented as, for example, ultraminiaturized sensors or 91 biophotonic transducers.

\section{RESULTS AND DISCUSSION}

Figure 2 shows atomic force microscopy (AFM) measurement $93 \mathrm{f} 2$ results of $33 \mathrm{~nm}$ thick EBL-structured OSTE resist. The 94 exposed patterns consisted of discs with equidistant diameter 95 and interdistance of 500 and $50 \mathrm{~nm}$, and of lines with 96 equidistant width and interdistance of 250, 100, 50, 30, and 2097 nm.

The line edge roughness (LER) increases with decreasing 99 lateral feature size. For feature interdistances of $250 \mathrm{~nm}$ or 100 higher, the structure height equals the full OSTE layer 101 thickness and the nonexposed resist is successfully removed 102 from the substrate surface. For smaller interdistances, the 103 structure height is reduced, although features as small as $20 \mathrm{~nm} 104$ remain easily distinguishable. These features are the smallest 105 and most dense reported for thiol-ene polymer networks, 106 regardless of nanopatterning method.

E-beam curing of thiol-ene systems can proceed via free 108 radical polymerization without the use of initiators. ${ }^{6,22} 109$ However, to achieve micrometer-scale or smaller resolution 110 requires the combination of a high thiol-ene off-stoichiometry 111 


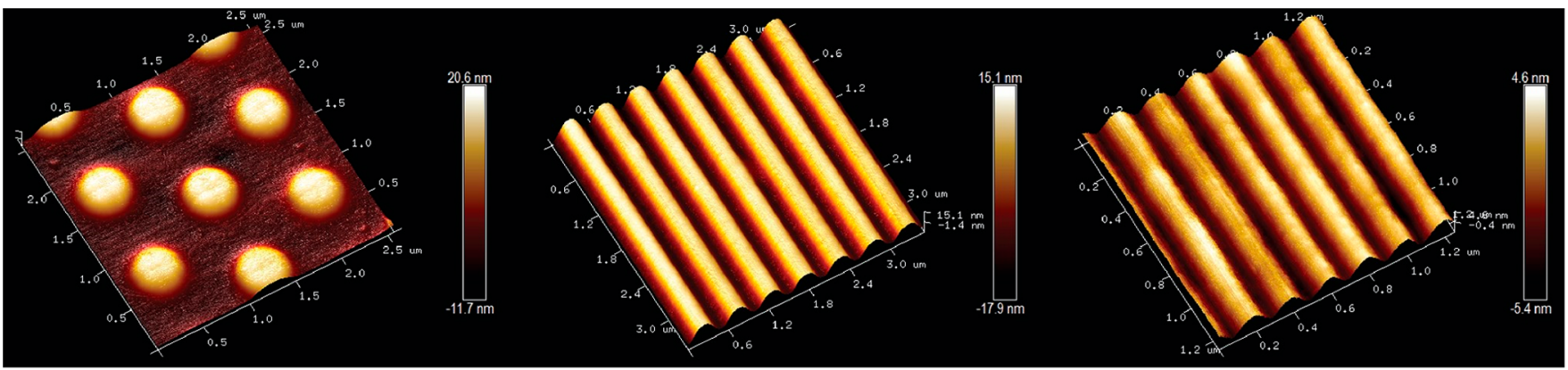

(a)

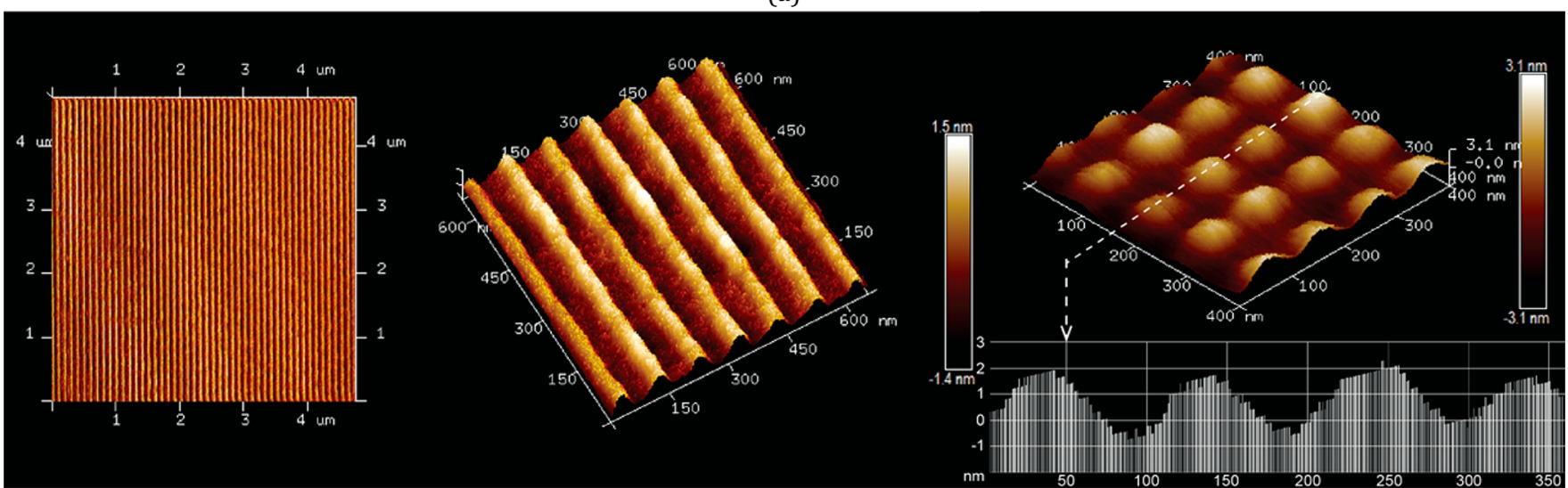

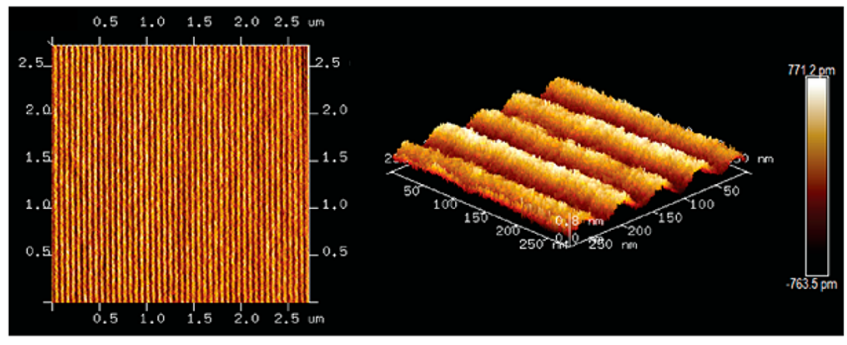

(c) (b)

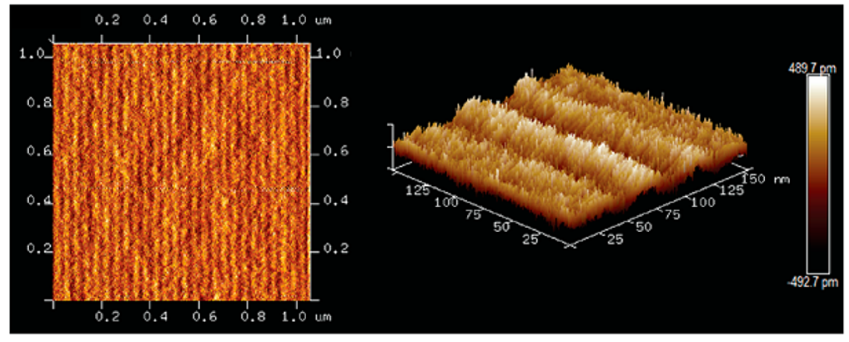

(d)

Figure 2. AFM images of gratings and mesa structures of different size, patterned in OSTE via EBL, in which the color bars represent the height of the structures. (a) Circular pillars of $500 \mathrm{~nm}$ diameter and half-pitch (left), gratings of $250 \mathrm{~nm}$ width and half-pitch (middle), and gratings of $100 \mathrm{~nm}$ width and half-pitch (right). (b) Gratings of $50 \mathrm{~nm}$ width and half-pitch (left) and circular pillars of $50 \mathrm{~nm}$ diameter and half-pitch (right). The dotted white line indicates where the cross-section lays. (c) Gratings of $30 \mathrm{~nm}$ width and half-pitch. (d) Gratings of 20 nm width and half-pitch. AFM, atomic force microscopy; EBL, e-beam lithography.

112 and the addition of inhibitor in the resist formulation. 113 Photostructuring of off-stoichiometric thiol-ene prepolymer 114 mixture depletes the deficient monomer in the nonexposed 115 region immediately adjacent to the exposed prepolymer, which 116 suppresses gelation caused by radical diffusion in that region. ${ }^{18}$ 117 We hypothesize that a similar effect occurs during e-beam 118 exposure. The addition of inhibitor compound further 119 prohibits the broadening of features by scavenging radicals in 120 nonexposed areas. The high feature density shown in Figure 2 121 confirms the prevention of feature broadening in the thiol-ene 122 network.

123 Figure 3 shows a light microscopy image of $5 \mu \mathrm{m}$ square 124 OSTE resist features after EBL exposure to doses between 100 125 and $2325 \mu \mathrm{C} / \mathrm{cm}^{2}$, followed by development, demonstrating 126 that OSTE EBL resist offers a wide dose window leading to a 127 wide process window, high robustness, and reproducibility. For 128 features of lateral size above $500 \mathrm{~nm}$, the demanded exposure 129 dose ranges from 100 to $500 \mu \mathrm{C} / \mathrm{cm}^{2}$. Smaller features require higher doses of $500-1000 \mu \mathrm{C} / \mathrm{cm}^{2}$, which is comparable to the 130 900-1000 $\mu \mathrm{C} / \mathrm{cm}^{2}$ dose needed for the widely used negative 131 tone resist HSQ.

132

Figure S1 shows XPS spectra and elemental states measured 133 on exposed and nonexposed areas of the e-beam resist after 134 development. For exposed areas, resonance peaks at the 135 characteristic binding energy of $163 \mathrm{eV}$ indicate that in average 136 $4.6 \%$ of the surface consists of thiol groups. ${ }^{26}$ We attribute the 137 relatively high nitrogen, high sulfur and low chromium content, 138 when compared to nonexposed areas, to the presence of the 139 thiol monomer $\mathrm{C}_{18} \mathrm{H}_{27} \mathrm{~N}_{3} \mathrm{O}_{9} \mathrm{~S}_{3}$, and the uncovering of the 140 underlying substrate during development.

The developed e-beam resist has an e-modulus of $3 \mathrm{GPa} 142$ (Figure S2). This is the highest reported stiffness for off- 143 stoichiometry thiol-enes, ${ }^{27}$ and substantially stiffer than the 144 respective 130 and $60 \mathrm{MPa}$ stiffness of similar stoichiometric 145 and off-stoichiometric thiol-ene formulations that were UV- 146 cured. 


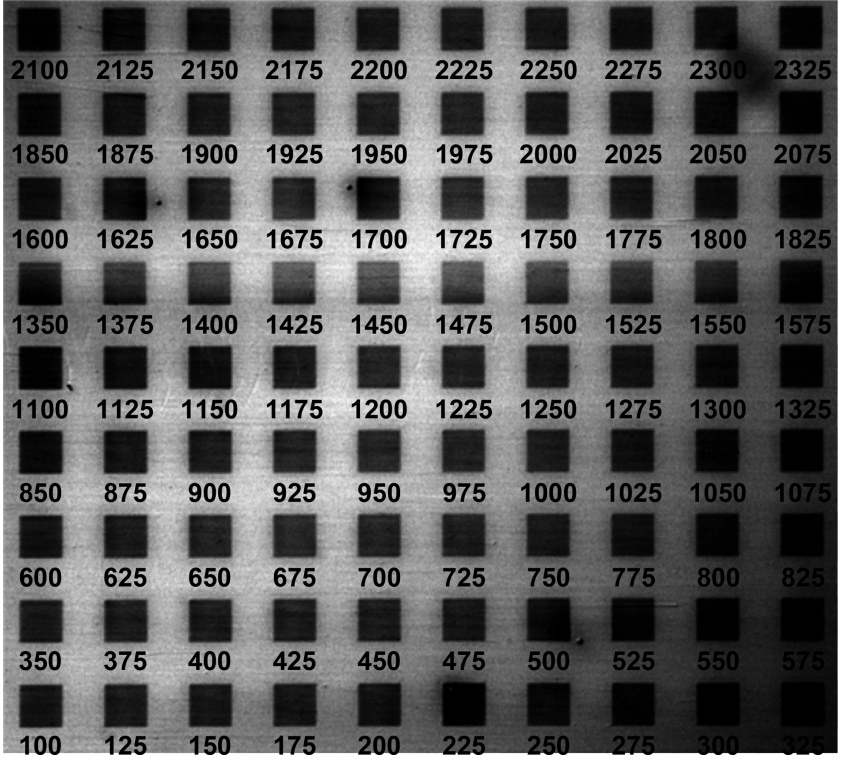

Figure 3. Light microscopy image of $5 \times 5 \mu \mathrm{m}^{2}$ squares of the OSTE resist after dose testing. The numbers indicate the exposure dose in $\mu \mathrm{C} / \mathrm{cm}^{2}$.
We speculate that the high degree of stiffening in the e-beam 148 versus UV cured formulations is due to cross-linking via 149 hydrogen abstraction and subsequent bond formation via 150 radical-radical coupling reactions.

Figure 4 shows fluorescence microscopy images of the $152 \mathrm{f} 4$ OSTE resist after EBL, followed by biofunctionalization with 153 biotin and streptavidin-Alexa Fluor 488 conjugate. The 154 exposed patterns consisted of a fractal tree that features a 155 $100 \mu \mathrm{m}$ wide trunk and twigs of width as small as $100 \mathrm{~nm} ; 156$ gratings and arrays of squares and discs of $10 \mu \mathrm{m}$ line size and 157 diameter; and line structures of $50 \mu \mathrm{m}$ length and 250, $500 \mathrm{~nm} 158$ width with interspacing of 2.5 and 25,1 and $50 \mu \mathrm{m}, 159$ respectively.

The fluorescence signal intensity from the OSTE structures 161 is $7.4 \times$ higher than that of the control surface (green channel, 162 analyzed by ImageJ). The high fluorescence intensity following 163 the biofunctionalization demonstrates that the thiol-ene resist 164 features chemically reactive thiols on its surface. Thiol reactive 165 groups enable diverse types of biofunctionalization, for 166 example, thiol-gold, thiol-ene, and thiol-Michael addition 167 (click) reactions, with the latter including thiol-maleimide, 168 thiol-vinyl sulfone, thiol-(meth)acrylate, and thiol-yne 169 interactions. $^{28}$ Here, biotin moieties bind directly and 170

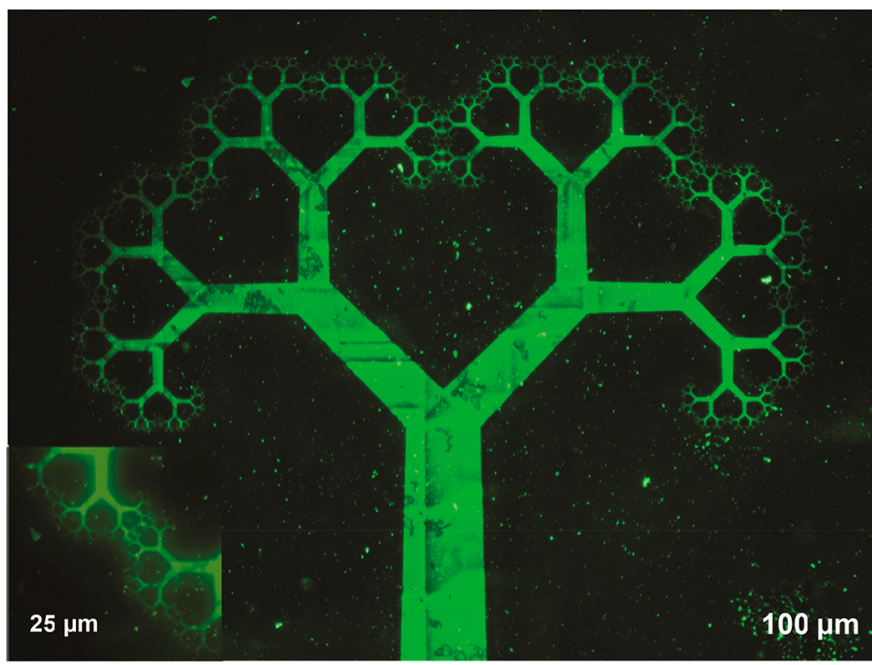

(a)

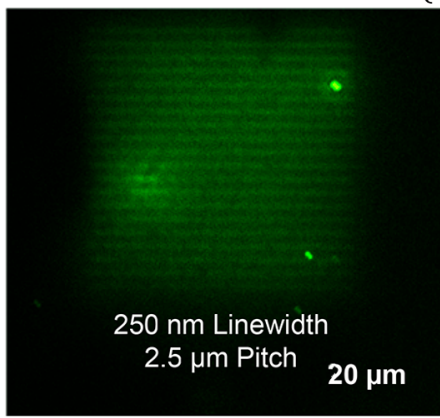

(c)

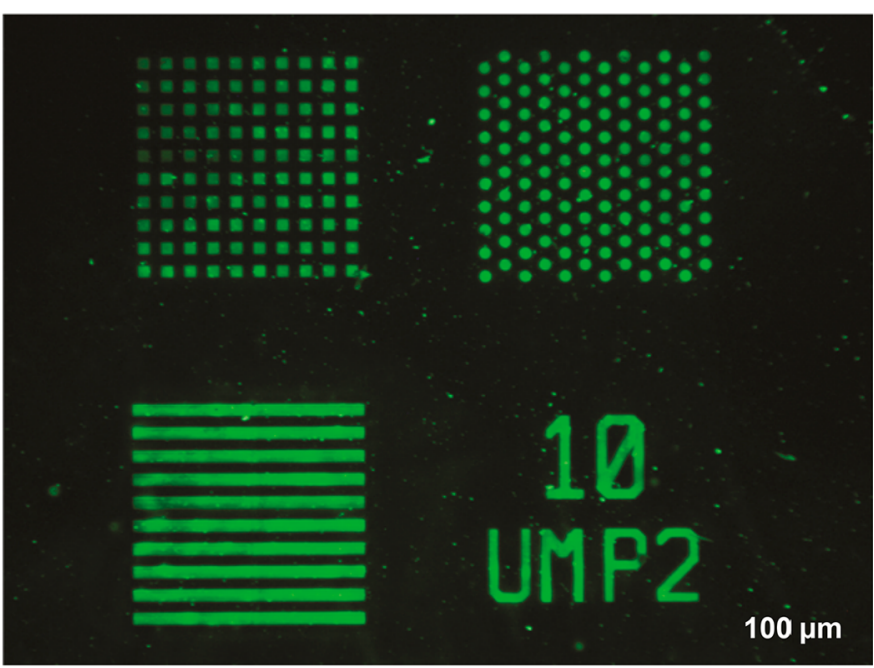

(b)

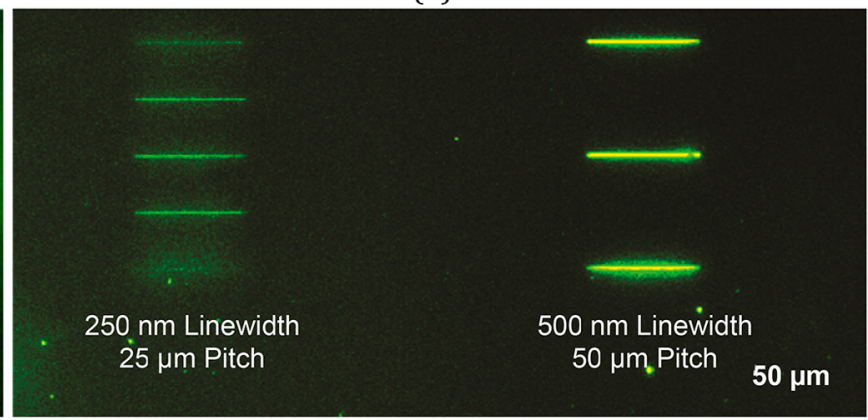

(e)

Figure 4. Fluorescence microscopy images of EBL nanopatterned OSTE structures of different size after incubation with biotin and fluorescently labeled streptavidin. (a) Fractal tree shape with microtrunk of width $100 \mu \mathrm{m}$ and branches as small as $100 \mathrm{~nm}$. (b) Dot and line structures size, line width, and interspacing of $10 \mu \mathrm{m}$. (c) Line structures of $50 \mu \mathrm{m}$ length, with respective width and pitch of $250 \mathrm{~nm}, 2.5$ $\mu \mathrm{m}$. (d) Line structures of $50 \mu \mathrm{m}$ length, with respective width and pitch of $500 \mathrm{~nm}, 1 \mu \mathrm{m}$. (e) Line structures of $50 \mu \mathrm{m}$ length with a respective width and pitch of, from left to right, $250 \mathrm{~nm}$ and $25 \mu \mathrm{m}$; and $500 \mathrm{~nm}$ and $50 \mu \mathrm{m}$. EBL, e-beam lithography; OSTE, offstoichiometric thiol-ene. 
171 covalently to the e-beam structured OSTE resist surface via a 172 spontaneous thiol-maleimide Michael addition reaction. The 173 subsequent attachment of streptavidin to the immobilized 174 biotin constitutes a full bioassay on top of the e-beam 175 patterned resist.

176 The lower limit to the protein patterned feature size in this 177 work, $250 \mathrm{~nm}$, is attributed to the requirement of a very fine 178 process window in the development procedure for dense 179 structures. Figure 2 shows the creation of smaller geometrical 180 features, albeit with reduced structural height. This leads us to 181 hypothesize that smaller protein features may be feasible 182 during EBL of thinner OSTE layers, with a thickness 183 comparable to the structural height variations obtained for 184 these smaller line width structures. In addition, tuning the 185 development process with regards to temperature, time and 186 solvent chemistry, along with using higher e-beam energies, 187 may enable more distinct sub-250 $\mathrm{nm}$ features.

188 Our approach has a number of advantages over previous 189 EBL-based in situ protein patterning techniques. We do not 190 expose proteins to the demanding processing conditions inside 191 the e-beam chamber, such as ultrahigh vacuum and high 192 electron energy, widening the possibility of EBL protein 193 patterning to more sensitive proteins. Moreover, our approach 194 obviates multistep surface modifications, such as building SAM 195 layers on top of an e-beam resist or on the underlying 196 substrate.

197 OSTE is also mechanically more robust, with an E-modulus $198 \sim 3 \mathrm{GPa}$, compared to the E-moduli of hydrogels, <100 kPa. 199 Due to the nature of thiol-click chemistry most processes can 200 be performed under benign conditions that are compatible 201 with proteins, nucleic acids, and cell cultures. This enables life202 science applications, such as the straightforward nanoscale 203 patterning of sensitive biomolecules and small footprint 204 bioassay immobilization.

205 We hypothesize that in a broader perspective, this work will 206 also enable combining other specific features of OSTE with 207 nanoscale structures, such as the use of thiol-click reactions for 208 direct metal coating, for example, via thiol-gold chemistry, ${ }^{29}$ 209 grafting of functional layers, ${ }^{30}$ layer bonding, ${ }^{31}$ or component 210 self-assembly; the tuning of the mechanical stiffness, where the 211 previous and current work show E-moduli in the range 2.8 $212 \mathrm{MPa}$ to $3 \mathrm{GPa}^{27,32}$ and the high and tunable refractive index. ${ }^{33}$ 213 The combination of these properties may further lead to 214 potential applications in nanoscale electronic, photonic and 215 robotic systems.

\section{CONCLUSIONS}

217 In conclusion, this work introduces off-stoichiometric thiol218 ene copolymers as a new class of e-beam resist. The native 219 surfaces, including the side walls, of the mechanically robust 220 resist structures act as sites for direct and covalent biomolecule 221 immobilization. The results presented here hold specific 222 promises for life-science applications. Combining these results 223 with other features of OSTE materials may further lead to 224 ultraminiaturized biohybrid electronic, photonic and robotic 225 systems.

\section{METHODS}

227 Substrates. As substrate materials, both silicon and fused silica 228 chips coated with $20 \mathrm{~nm}$ chromium were used. The thin $\mathrm{Cr}$ 229 conductive layer reduces electron charge accumulation in the resist 230 while minimizing the electron backscattering and its subsequent 231 proximity effect.
Preparation of Thiol-Ene Based Precursor Formulations. 232 (1) The OSTE EBL resist was prepared in three steps. First, $83.2 \% 233$ $\mathrm{wt} / \mathrm{wt}$ of the trifunctional thiol monomer tris[2-(3-234 mercaptopropionyloxy)ethyl] isocyanurate (BOC Sciences) and 235 $16.8 \% \mathrm{wt} / \mathrm{wt}$ of the tetra-functional allyl monomer glyoxal bis(diallyl 236 acetal) (Sigma-Aldrich, Germany) were thoroughly mixed using a 237 speed-mixer (Hauschild Engineering, Germany), resulting in a 238 monomer composition with functional group molar ratio trithiol:te- 239 tra-allyl of 1.8:1. Second, the thiol-ene resist was diluted in propylene 240 glycol monomethyl ether acetate (Sigma-Aldrich, Germany) to create 241 a solution of concentration $5 \% \mathrm{wt} / \mathrm{wt}$. Finally, $0.67 \% \mathrm{wt} / \mathrm{wt}$ of an 242 inhibitor solution was added, consisting of $15 \% \mathrm{wt} / \mathrm{wt}$ of Q-1301 243 (Wako Chemical Inc.) dissolved in tetrahydrofuran (Sigma-Aldrich, 244 Germany). The OSTE EBL resist solution was stored in a dark 245 environment at $+4{ }^{\circ} \mathrm{C}$ until used.

246

(2) A new precursor formulation of OSTE for UV curing purposes 247 was prepared by addition of $0.5 \% \mathrm{wt} / \mathrm{wt}$ of 1-hydroxycyclohexyl 248 phenyl ketone (IRG) photoinitiator to the same 1.8:1 thiol-ene 249 blend and dilution in the same solvent to obtain a final 5\% wt/wt 250 thiol-ene solution.

(3) An on-stoichiometric thiol-ene (ONSTE) formulation for UV 252 curing was prepared by $73.4 \% \mathrm{wt} / \mathrm{wt}$ of the thiolmonomer mixed with 253 $26.6 \% \mathrm{wt} / \mathrm{wt}$ of the allyl monomer. The resulting 1:1 functional group 254 ratio blend was mixed with $0.5 \% \mathrm{wt} / \mathrm{wt}$ of the photoinitiator and 255 diluted in the solvent to reach a 5\% wt/wt thiol-ene solution. 256

The thiol-ene based solutions were stored in a dark environment 257 at $+4{ }^{\circ} \mathrm{C}$ until used.

258

Substrate Preparation by Spin-Coating. Thin films of thiol- 259 ene based resists were spin-coated on the substrates at $4000 \mathrm{rpm}$, with 260 an acceleration of $3000 \mathrm{rpm} / \mathrm{s}$, for $5 \mathrm{~s}$ resulting in a 30-50 nm resist 261 thickness, as measured by using a stylus profiler (KLA-Tencor P-15, 262 Milpitas). The high spin acceleration rate was used to ensure a 263 uniform film coating of OSTE.

E-Beam Patterning. E-beam writing was performed (Raith 150, 265 Germany) at an exposure voltage of $25 \mathrm{keV}$. Different exposure doses 266 in the range $100-2325 \mu \mathrm{C} / \mathrm{cm}^{2}$ were applied, depending on the 267 targeted resolution.

Different features were e-beam patterned, including gratings and 269 arrays of circular and square pillars with feature size between $20 \mathrm{~nm} 270$ and $10 \mu \mathrm{m}$. We also patterned a single fractal structure containing 271 features of size ranging from $100 \mathrm{~nm}$ to $100 \mu \mathrm{m}$.

UV Curing. The precursors adapted for UV curing were exposed 273 to collimated ( $3^{\circ}$ collimation half angle) near UV short-arc mercury 274 lamp (OAI, Milpitas) at $10.5 \mathrm{~mW} \mathrm{~cm} \mathrm{~cm}^{-2}$ for $10 \mathrm{~S}$.

Development. Samples were developed by immersion into hexyl 276 acetate (Sigma-aldrich, Germany) for $30 \mathrm{~s}$ at room temperature. 277

XPS Analysis. To measure the relative concentration of the thiol 278 functional group next to the e-beam exposure, an e-beam structured 279 chip containing OSTE patterns was characterized by XPS 280 (VersaProbeIII Scanning XPS microprobe, monochromatic Al K $\alpha 281$ source $(h v=1486.6 \mathrm{eV})$, Beam size: $50 \mu \mathrm{m})$. Five positions on both 282 e-beam structured and nonexposed areas were selected for XPS 283 analysis (see Figure S2).

Protein Functionalization. A solution of $10 \mathrm{mM}$ maleimide- 285 PEG2-biotin in PBS (ThermoFisher, Sweden) was incubated on the 286 structured OSTE surface for $10 \mathrm{~min}$, followed by rinsing with PBS 287 containing $0.05 \%$ Tween 20 (VWR Chemicals, Sweden). Thereafter, 288 streptavidin solution (Alexa Fluor488 conjugate, $12 \mu \mathrm{M}$ in PBS, 289 ThermoFisher, Sweden) was incubated on the sample for $10 \mathrm{~min}, 290$ followed by rinsing with PBS containing $0.05 \%$ Tween 20 and pure 291 PBS in sequence, and drying at room temperature.

292

Microscopy. Structures were evaluated by light microscopy, 293 atomic force microscopy (AFM, from Bruker ICON) using 294 noncontact mode, and fluorescence microscopy (Nikon, Japan). 295

Elastic Modulus Analysis. Two samples entirely coated by thin 296 films of UV cured on-stoichiometric thiol-ene and OSTE 80\%, along 297 with an additional sample containing e-beam structured patterns of 298 OSTE $80 \%$ were quantitatively inspected using the same AFM 299 microscopy equipped with a multifrequency lock-in amplifier (MLA 300 hardware) and Intermodulation AFM software suite (Intermodulation 301 
302 Products $\mathrm{AB}$, Sweden). E-modulus quantification was done through 303 DMT-EXP model. ${ }^{34}$

\section{ASSOCIATED CONTENT}

\section{S Supporting Information}

306 The Supporting Information is available free of charge on the 307 ACS Publications website at DOI: 10.1021/acsnano.8b03709.

308

XPS analysis data and intermodulation AFM analysis

309 data (PDF)

\section{AUTHOR INFORMATION}

\section{Corresponding Author}

312 *E-mail: wouter@kth.se.

\section{ORCID}

314 Reza Zandi Shafagh: 0000-0003-4322-6192

315 Alexander Vastesson: 0000-0001-9651-4900

316 Wouter van der Wijngaart: 0000-0001-8248-6670

317 Notes

318 The authors declare no competing financial interest.

\section{REFERENCES}

320 (1) Freudenberg, U.; Liang, Y.; Kiick, K. L.; Werner, C. 321 Glycosaminoglycan-based Biohybrid Hydrogels: A Sweet and Smart 322 Choice for Multifunctional Biomaterials. Adv. Mater. 2016, 28, 88613238891 .

324 (2) Yoon, J.; Eyster, T. W.; Misra, A. C.; Lahann, J. Cardiomyocyte325 driven Actuation in Biohybrid Microcylinders. Adv. Mater. 2015, 27, 326 4509-4515.

327 (3) Rundqvist, J.; Mendoza, B.; Werbin, J. L.; Heinz, W. F.; 328 Lemmon, C.; Romer, L. H.; Haviland, D. B.; Hoh, J. H. High Fidelity 329 Functional Patterns of an Extracellular Matrix Protein by Electron 330 Beam-based Inactivation. J. Am. Chem. Soc. 2007, 129, 59-67.

331 (4) Kolodziej, C. M.; Maynard, H. D. Electron-beam Lithography 332 for Patterning Biomolecules at the Micron and Nanometer Scale. 333 Chem. Mater. 2012, 24, 774-780.

334 (5) Bat, E.; Lee, J.; Lau, U. Y.; Maynard, H. D. Trehalose 335 Glycopolymer Resists Allow Direct Writing of Protein Patterns by 336 Electron-beam Lithography. Nat. Commun. 2015, 6, 6654.

337 (6) Hoyle, C. E.; Lee, T. Y.; Roper, T. Thiolenes: Chemistry of the 338 past with Promise for the Future. J. Polym. Sci., Part A: Polym. Chem. 339 2004, 42, 5301-5338.

340 (7) Ashley, J. F.; Cramer, N. B.; Davis, R. H.; Bowman, C. N. Soft341 lithography Fabrication of Microfluidic Features Using Thiol-ene 342 Formulations. Lab Chip 2011, 11, 2772-2778.

343 (8) Hagberg, E. C.; Malkoch, M.; Ling, Y.; Hawker, C. J.; Carter, K. 344 R. Effects of Modulus and Surface Chemistry of Thiol-ene 345 Photopolymers in Nanoimprinting. Nano Lett. 2007, 7, 233-237.

346 (9) Campos, L. M.; Meinel, I.; Guino, R. G.; Schierhorn, M.; Gupta, 347 N.; Stucky, G. D.; Hawker, C. J. Highly Versatile and Robust 348 Materials for Soft Imprint Lithography Based on Thiol-ene Click 349 Chemistry. Adv. Mater. 2008, 20, 3728-3733.

350 (10) Khire, V. S.; Yi, Y.; Clark, N. A.; Bowman, C. N. Formation and 351 Surface Modification of Nanopatterned Thiol-ene Substrates Using 352 Step and Flash Imprint Lithography. Adv. Mater. 2008, 20, 33083533313 .

354 (11) Lin, H.; Wan, X.; Jiang, X.; Wang, Q.; Yin, J. a Nanoimprint 355 Lithography Hybrid Photoresist Based on the Thiolene System. Adv. 356 Funct. Mater. 2011, 21, 2960-2967.

357 (12) Ahn, S. H.; Guo, L. J. Large-area Roll-to-roll and Roll-to-plate 358 Nanoimprint Lithography: A Step Toward High-throughput 359 Application of Continuous Nanoimprinting. ACS Nano 2009, 3, $3602304-2310$.

361 (13) Malloy, M.; Litt, L. C. Technology Review and Assessment of 362 Nanoimprint Lithography for Semiconductor and Patterned Media 363 Manufacturing. J. Micro/Nanolithogr., MEMS, MOEMS 2011, 10, 364032001.
(14) Carlborg, C. F.; Haraldsson, T.; Oberg, K.; Malkoch, M.; Van 365 Der Wijngaart, W. Beyond Pdms: Off-stoichiometry Thiol-ene (Oste) 366 Based Soft Lithography for Rapid Prototyping of Microfluidic 367 Devices. Lab Chip 2011, 11, 3136-3147. 368

(15) Lafleur, J. P.; Kwapiszewski, R.; Jensen, T. G.; Kutter, J. P. 369 Rapid Photochemical Surface Patterning of Proteins in Thiol-ene 370 Based Microfluidic Devices. Analyst 2013, 138, 845-849. 371

(16) Ejserholm, F.; Stegmayr, J.; Bauer, P.; Johansson, F.; Wallman, 372 L.; Bengtsson, M.; Oredsson, S. Biocompatibility of a Polymer Based 373 on Off-stoichiometry Thiol-enes+Epoxy (Oste+) for Neural Implants. 374 Biomater. Res. 2015, 19, 19.

(17) Sticker, D.; Rothbauer, M.; Lechner, S.; Hehenberger, M.-T.; 376 Ertl, P. Multi-layered, Membrane-integrated Microfluidics Based on 377 Replica Molding of a Thiol-ene Epoxy Thermoset for Organ-on-a- 378 chip Applications. Lab Chip 2015, 15, 4542-4554. 379

(18) Hillmering, M.; Pardon, G.; Vastesson, A.; Supekar, O.; 380 Carlborg, C. F.; Brandner, B. D.; Van Der Wijngaart, W.; Haraldsson, 381 T. Off-stoichiometry Improves the Photostructuring of Thiolenes 382 Through Diffusion-induced Monomer Depletion. Microsyst. Nanoeng. 383 2016, 2, 15043.

(19) Hoyle, C. E.; Lowe, A. B.; Bowman, C. N. Thiol-click 385 Chemistry: A Multifaceted Toolbox for Small Molecule and Polymer 386 Synthesis. Chem. Soc. Rev. 2010, 39, 1355-1387.

(20) Hoffmann, C.; Pinelo, M.; Woodley, J. M.; Daugaard, A. E. 388 Development of a Thiolene Based Screening Platform for Enzyme 389 Immobilization Demonstrated Using Horseradish Peroxidase. Bio- 390 technol. Prog. 2017, 33, 1267-1277.

(21) Groschel, A. H.; Muller, A. H. E. Self-assembly Concepts for 392 Multicompartment Nanostructures. Nanoscale 2015, 7, 11841- 393 11876 394

(22) Hoyle, C. E.; Bowman, C. N. Thiolene Click Chemistry. Angew. 395 Chem., Int. Ed. 2010, 49, 1540-1573. 396

(23) Kwak, M.; Herrmann, A. Nucleic Acid/Organic Polymer 397 Hybrid Materials: Synthesis, Superstructures, and Applications. 398 Angew. Chem., Int. Ed. 2010, 49, 8574-8587.

(24) Sawicki, L. A.; Kloxin, A. M. Design of Thiol-ene Photoclick 400 Hydrogels Using Facile Techniques for Cell Culture Applications. 401 Biomater. Sci. 2014, 2, 1612-1626.

(25) Sticker, D.; Lechner, S.; Jungreuthmayer, C.; Zanghellini, J.; 403 Ertl, P. Microfluidic Migration and Wound Healing Assay Based on 404 Mechanically Induced Injuries of Defined and Highly Reproducible 405 Areas. Anal. Chem. 2017, 89, 2326-2333.

(26) Wagner, C. D.; Riggs, W. M.; Davis, L. E.; Moulder, J.; 407 Muilenberg, G. E. Handbook of X-ray Photoelectron Spectroscopy: A 408 Reference Book of Standard Data for Use in X-ray Photoelectron 409 Spectroscopy; Perkin-Elmer Corp.: Eden Prairie, MN, 1979.

(27) Sandström, N.; Shafagh, R. Z.; Vastesson, A.; Carlborg, C. F.; 411 van der Wijngaart, W.; Haraldsson, T. Reaction Injection Molding 412 and Direct Covalent Bonding of Oste+ Polymer Microfluidic Devices. 413 J. Micromech. Microeng. 2015, 25, 075002.

(28) Nair, D. P.; Podgorski, M.; Chatani, S.; Gong, T.; Xi, W.; 415 Fenoli, C. R.; Bowman, C. N. the Thiol-michael Addition Click 416 Reaction: A Powerful and Widely Used Tool in Materials Chemistry. 417 Chem. Mater. 2014, 26, 724-744.

418

(29) Rahiminejad, S.; Hansson, J.; Köhler, E.; van der Wijngaart, W.; 419 Haraldsson, T.; Haasl, S.; Enoksson, P. Rapid Manufacturing of 420 OSTE Polymer RF-MEMS Components. Proceedings of the IEEE 30th 421 International Conference on Microelectromechanical Systems (MEMS) 422 2017, 901-904.

(30) Pardon, G.; Saharil, F.; Karlsson, J. M.; Supekar, O.; Carlborg, 424 C. F.; van der Wijngaart, W.; Haraldsson, T. Rapid Moldfree 425 Manufacturing of Microfluidic Devices with Robust and Spatially 426 Directed Surface Modifications. Microfluid. Nanofluid. 2014, 17, 773- 427 779.

(31) Saharil, F.; Carlborg, C. F.; Haraldsson, T.; Van Der Wijngaart, 429 W. Biocompatible "Click" Wafer Bonding for Microfluidic Devices. 430 Lab Chip 2012, 12, 3032-3035.

(32) Hansson, J.; Karlsson, J. M.; Carlborg, C. F.; van der Wijngaart, 432 W.; Haraldsson, T. Low Gas Permeable and Non-absorbent Rubbery 433 
434 OSTE For Pneumatic Microvalves. Proceedings of the IEEE 27th 435 International Conference on Microelectromechanical Systems (MEMS) 436 2014, 987-990.

437 (33) Hata, E.; Tomita, Y. Stoichiometric Thiol-to-ene Ratio 438 Dependences of Refractive Index Modulation and Shrinkage of 439 Volume Gratings Recorded in Photopolymerizable Nanoparticle440 polymer Composites Based Onstep-growth Polymerization. Opt. 441 Mater. Express 2011, 1, 1113-1120.

442 (34) Forchheimer, D.; Platz, D.; Tholen, E. a.; Haviland, D. B. 443 Model-based Extraction of Material Properties in Multifrequency 444 Atomic Force Microscopy. Phys. Rev. B: Condens. Matter Mater. Phys. 445 2012, 85, 195449. 